\title{
Online Voltage Stability Monitoring and Control Using Limited Synchrophasor Measurements
}

\author{
Ruoxi Zhu \\ Thesis submitted to the Faculty of the \\ Master of Science \\ in \\ Electrical Engineering \\ Chen-Ching Liu, Chair \\ Virgilio A. Centeno \\ Jaime De La Reelopez
}

Virginia Polytechnic Institute and State University

in partial fulfillment of the requirements for the degree of

December 16, 2019

Blacksburg, Virginia

Keywords: PMU, Voltage stability, OLTC control

Copyright 2020, Ruoxi Zhu 


\section{Online Voltage Stability Monitoring and Control Using Limited Synchrophasor Measurements}

Ruoxi Zhu

(ABSTRACT)

As the scale and complexity of an interconnected power grid has increased significantly, power systems can be operated close to the verge of voltage instability. With the application of Phasor Measurement Units (PMUs), dispatchers are able to monitor long term voltage stability in a real time operational environment. This research addresses the critical issues by proposing three different methods. Voltage Stability Assessment Index (VSAI) is a Thévenin Equivalent (TE) based method considering voltage dynamic mechanisms. To extend the model from one load bus to a critical load center, Optimal Power Flow-Loading limit (OPF-LI) is developed to assess the voltage stability margin. To utilize limited available PMU measurements, State Calculator (SC) is included in the algorithm to approximate the dynamic states at the buses where PMU measurements are not available. The online voltage regulating method in terms of On-load Tap Changer (OLTC) control is also investigated. The methods proposed in this research have been validated with the test cases from the WECC 179 bus system. 


\section{Online Voltage Stability Monitoring and Control Using Limited Synchrophasor Measurements}

Ruoxi Zhu

(GENERAL AUDIENCE ABSTRACT)

This thesis proposed a hybrid solution of voltage stability monitoring and control in a power system. For the performance of motors, heaters or other loads in the power system, it is important that the customers are supplied with stable voltage. The variation of the voltage may cause damages to the load. Therefore, the methods in this thesis provides a feasible solution to monitor voltage stability of load centers in a power system. In addition, a novel approach for voltage control is proposed to prevent a voltage collapse of the system. The simulation results illustrate that the approach introduced in this thesis is promising for real time application. 


\section{Acknowledgments}

This work was funded through Project S-65 of Power Systems Engineering Research Cen-

ter (PSERC).Most of the work was completed while the author was at Washington State University. 


\section{Contents}

List of Figures vii

List of Tables viii

1 Introduction $\quad 1$

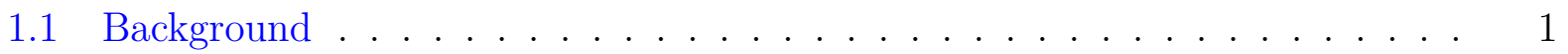

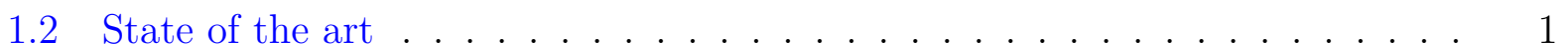

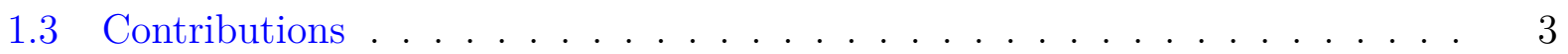

1.4 Thesis Organization . . . . . . . . . . . . . . . . . 3

2 Voltage Stability Assessment Index 4

2.1 Formulation of VSAI . . . . . . . . . . . . . . . . 4

2.2 VSAI with voltage dynamic mechanism . . . . . . . . . . . 5

$\begin{array}{llr}3 & \text { OPF-LI with State Calculator } & 8\end{array}$

3.1 Ward-PV model . . . . . . . . . . . . . . . . . 8

$3.2 \mathrm{OPF}$ based algorithm . . . . . . . . . . . . . . . 10

3.3 OPF-LI with State Calculator . . . . . . . . . . . . . . 11

4 OLTC operating control 13 
5 Simulation Results $\quad 16$

5.1 Comparison of VSAI-I and VSAI-II . . . . . . . . . . . . . . 18

5.2 Validation of OPF-LI with TSAT . . . . . . . . . . . . . . 20

5.3 OPF-LI with $\mathrm{SC} \ldots \ldots \ldots \ldots \ldots$

5.3.1 Case $1 \ldots \ldots \ldots \ldots \ldots$

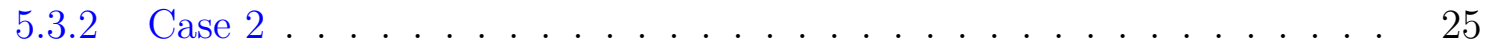

5.4 OLTC operating control . . . . . . . . . . . . . . . . . . . 26

6 Conclusions and Future Works 29

$\begin{array}{lr}\text { Bibliography } & 30\end{array}$ 


\section{List of Figures}

3.1 Ward-PV model . . . . . . . . . . . . . . . . . . . . . . . 9 9

3.2 The flowchart of State Calculator $(\mathrm{SC}) \ldots \ldots \ldots 11$

5.1 Area 1 of WECC 179 bus system . . . . . . . . . . . . . . . 17

5.2 VSAI and voltage magnitudes of IEEE 30-bus system after a contingency . . 18

5.3 VSAI-I of the load buses $\ldots \ldots \ldots \ldots$

5.4 VSAI-II of the load buses $\ldots \ldots \ldots \ldots$

5.5 System states (Bus voltage and angle) in Arealincreased to1.25 or 1.26 . . 21

5.6 State calculator results - Case $1 \ldots \ldots \ldots 24$

5.7 The loading limit during the contingency-Case $1 \ldots \ldots 24$

5.8 State calculator results-Case $2 \ldots \ldots \ldots \ldots \ldots$

5.9 The loading limit during the contingency-Case $2 \ldots \ldots 26$

5.10 The comparison of voltage profiles - without OLTC blocking control . . . . 27

5.11 The comparison of voltage profiles - with OLTC blocking control . . . . . 27 


\section{List of Tables}

5.1 Results of OPF-LI. . . . . . . . . . . . . . . . . . . . . . . . 22

5.2 PMU location . . . . . . . . . . . . . . . . . . . . . . 23 


\section{List of Abbreviations}

OLTC On-Load Tap Changer

OPF Optimal Power Flow

PMU Phasor Measurement Unit

SC State Calculator

TE Thévenin Equivalent

VSAI Voltage Stability Assessment Index 


\section{Chapter 1}

\section{Introduction}

\section{$1.1 \quad$ Background}

Because of the significant expansion of the interconnected power grid, major blackouts in power systems have occurred due to system instability. Long term voltage stability is a focus area for power system research.

Since power systems can be operated close to the verge of voltage instability, it is necessary for dispatchers to monitor voltage stability in an on-line operating condition. A voltage collapse is characterized by the fact that the voltage magnitude of some buses decline over time. Many algorithms have been proposed to estimate the margin to voltage instability at an operation point.

With a limited number of phasor measurements, a methodology for long term voltage stability monitoring using a reduced network is introduce in this thesis.

\subsection{State of the art}

Long term voltage stability refers to the stability problem that occurs over a relatively long time frame, such as several minutes. This kind of stability problem usually involves slow acting devices such as thermostatically controlled loads, LTC transformers, and distribution voltage regulators. 
To assess the long term voltage stability for a power system, two classes of methods have been developed. One is a model based method. According to the system topology and operation condition, several indices can be extracted from power flow equations. Based on the Jacobian matrix, several modes can be derived from the eigenvalues. Once the critical mode becomes zero, the system is considered to be unstable [11]. For the detection of the unstable condition, continuous power flow method, bifurcation theory and sensitivity methods are proposed [1],[5],[8].

The other class, which is also popular is the measurements based method. With numerous measurements installed through the smart grid projects, it is feasible to efficiently monitor the dynamic voltage behavior of the bulk power system. The Phasor Measurement Unit (PMU) technology enables the measurement-based methods for voltage stability monitoring and control. Taking advantage of PMU measurements, this thesis presents the strategies for real time monitoring and control of voltage stability.

A number of applications have been developed based on the utilization of PMU measurements. It becomes practical to use phasor measurements to detect voltage instability in power system. Comparing with the conventional model-based approaches, PMU technology improves the computational speed for wide-area situational awareness [2]. Before PMUs are widely deployed in the system, the TE based methods, e.g., VIP method estimates the voltage stability margin by using local measurements at one location [12]. In recent years, with PMU measurements available at multiple locations, real-time voltage stability monitoring has been proposed using various algorithms, such as QV indices, channel components transform, and regression tree $[4,10,16]$. Instead of using only local measurements, PMU data provide a systematic approach to assess the voltage stability margin [7]. Some methods use PMUs to detect voltage instability [6]. However, regarding online voltage stability monitoring, most methods use the steady state operating point and neglect dynamic mechanisms of voltage stability. 


\subsection{Contributions}

The contribution of the proposed methods in this thesis is to incorporate dynamic behaviors into the computational model for voltage stability monitoring, enhancing the accuracy of voltage stability indices. Furthermore, considering the impact of OLTC control for voltage stability, an online OLTC control strategy is proposed. To deal with the incomplete observability of PMU measurements, a novel approach is proposed to estimate voltage stability margin with limited PMU measurements in a real time environment.

\subsection{Thesis Organization}

The remaining of the thesis is organized as follows: Chapter 2 provides the formulation of voltage stability assessment index (VSAI) method considering voltage dynamic mechanisms. In Chapter 3, to monitor voltage stability of a load area, OPF-LI with PMU measurements including unavailable states estimated by State Calculator (SC) is described. Then, OLTC operating control is discussed in Chapter 4. Simulation results are summarized in Chapter 5. Finally, the conclusion is presented in Chapter 6 . 


\section{Chapter 2}

\section{Voltage Stability Assessment Index}

\subsection{Formulation of VSAI}

The existing centralized model based approaches for voltage stability monitoring often use multiple power flows to compute the voltage stability margin of a system. Hence it is computationally burdensome and are not suitable for real time applications.

The VSAI algorithm is a hybrid approach using the PMU data and network information to

calculate voltage stability indices at each load bus. VSAI, as a TE based method, computes the Thevenin impedance at each load bus without iterative calculations.

The Jacobian matrix is obtained based on the topological data and system parameters. Then, two sets of additional system characteristics (e.g., estimated voltages and currents for all buses) are calculated by (2.1) based on the specified perturbations at each load bus.

$$
\left[\begin{array}{c}
\Delta \theta_{i} \\
\Delta V_{i}
\end{array}\right]=\left[J^{-1}\right]\left[\begin{array}{c}
\Delta P_{i} \\
\Delta Q_{i}
\end{array}\right]
$$

where $\Delta P_{i}, \Delta Q_{i}$ denotes the specified perturbations, $\Delta \theta_{i}, \Delta V_{i}$ are the estimated voltage change, and $J$ denotes the Jacobian matrix. Finally, the Thevenin impedance is estimated based on the actual system parameters and the derived system parameters. Then VSAI is 
computed at load bus $k$, i.e.,

$$
V S A I_{k}=\frac{\mid \text { Average }\left[\frac{v_{k}-v_{k(+v e)}}{i_{k(+v e)-i_{k}}}, \frac{v_{k(-v e)-v_{k}}}{i_{k}-i_{k(-v e)}}\right] \mid}{\left|\frac{v_{k}}{i_{k}}\right|}
$$

where ' $k$ ' means the load bus $k$, the average value in (2.2) is the Thevenin impedance, and $\left|\frac{v_{k}}{i_{k}}\right|$ represents the load impedance based on the actual PMU data [3]. The estimated Thevenin impedance is compared with the load impedance in (2.2). Thus, $0<V S A I_{k}<1$ for any load bus $k$. $V S A I_{k}$ value approach "1" indicates that bus ' $k$ ' is close to the verge of the voltage instability. However, the original algorithm of $V S A I[3]$ without any operating limits may provide overly optimistic margins.

\subsection{VSAI with voltage dynamic mechanism}

To improve the accuracy of $V S A I$ for real-time environment, the enhanced version of $V S A I, V S A I-I I$, is proposed. It incorporates the voltage dynamic mechanism, such as generator excitation capability, stator current limit, and OLTCs [9, 13].

Under normal operating conditions, the terminal voltage of generator is constant value $V_{0}$ with voltage source $E$ and stator current $I$ are subject to upper limit $E_{\text {lim }}, I_{\text {lim }}$ respectively. The proposed techniques are as follows.

(1) Normal operation: $V=V_{0}, \quad E<E_{\text {lim }}, \quad I<I_{\text {lim }}$.

(2) Operation at the excitation voltage limit:

$$
P_{i}^{2}+\left[Q_{i}+\frac{V_{i}^{2}}{x_{d}}\right]^{2}=\frac{E_{i}^{2} V_{i}^{2}}{x_{d}^{2}} \geq \frac{E_{l i m}^{2} V_{i}^{2}}{x_{d}^{2}}
$$

When (2.3) is satisfied at any generator bus $i$, this bus violates the excitation voltage constraint. To model this scenario, a virtual PV node bus $i+1$ is added at bus $i$. The voltage 
magnitude of bus $i+1$ is specified as $E_{\text {lim }}$ as the voltage source. Meanwhile, bus $i$ is converted to a constant $\mathrm{PQ}$ bus. The load connected to bus $i$ determines $\mathrm{P}$ and $\mathrm{Q}$ of this new PQ bus. Furthermore, the admittance matrix is updated based on the change of the system topology and the power flow equations are modified as follows:

Bus $i+1$ (PV bus):

$$
P_{i+1}=E_{i+1} V_{i}\left(G_{(i+1) i} \cos \delta_{(i+1) i}+B_{(i+1) i} \sin \delta_{(i+1) i}\right)
$$

Bus $i$ (PQ bus):

$$
\begin{aligned}
P_{i} & =V_{i} \sum_{j=1}^{n+1} V_{j}\left(G_{i j} \cos \delta_{i j}+B_{i j} \sin \delta_{i j}\right) \\
Q_{i} & =V_{i} \sum_{j=1}^{n+1} V_{j}\left(G_{i j} \sin \delta_{i j}-B_{i j} \cos \delta_{i j}\right)
\end{aligned}
$$

where $i+1$ means that the number of buses increases considering the virtual bus. $V S A I$ is calculated with modified Jacobian matrix.

(3) Operation at the stator current limit:

$$
\frac{P_{i}^{2}+Q_{i}^{2}}{V_{i}^{2}}=I_{i}^{2} \geq I_{l i m}^{2}
$$

When (2.7) is satisfied at any generator bus $i$, this bus violates the stator current constraint. To model this scenario, bus $i$ is converted to a constant current source.

Considering the constant current source in the system, the power flow equation is presented as:

$$
\left(\frac{P_{i g}+Q_{i g}}{\widetilde{V}_{i}}\right)^{*}-\left(\frac{P_{i l}+Q_{i l}}{\widetilde{V}_{i}}\right)^{*}=Y_{i i} \widetilde{V}_{i}+\sum Y_{i j} \widetilde{V}_{j}
$$


where $P_{i g}, Q_{i g}$ are the active and reactive power output of bus $i$, and $P_{i l}, Q_{i l}$ are the load connected to bus $i$. Hence, if bus $i$ is converted to a constant current source, the equation for bus $i$ is updated by (2.9) :

$$
{\widetilde{I_{l i m}}}^{*}-\left(\frac{P_{i l}+Q_{i l}}{\widetilde{V}_{i}}\right)^{*}=Y_{i i} \widetilde{V}_{i}+\sum Y_{i j} \widetilde{V}_{j}
$$

Equation (2.8) is the main equation for the iterative algorithm. By using Gauss-Seidel method, voltage magnitude and angle are calculated at each bus. Then VSAI calculation is initialized by substituting the updated power flow results into the Jacobian matrix.

(4) OLTCs: The automation action of OLTC is based on the predefined voltage $V_{0}$ as the reference. The discrete model of OLTC [9] is given as follows:

$$
\begin{aligned}
& n_{k+1}=n_{k}-d \cdot f\left(V_{k}-V_{0}\right) \\
& f(x)=\left\{\begin{array}{cc}
-1 & \text { if } x \leq-\Delta v \\
0 & \text { if }|x|<\Delta v \\
1 & \text { if } x \geq \Delta v
\end{array}\right\}
\end{aligned}
$$

Note that the admittance matrix should be modified once the tap ratio is updated. Therefore, the Jacobian matrix is updated correspondingly.

Considering the voltage dynamic mechanisms, the state variables change with time. Hence, relative to the steady state model, the proposed method is more accurate in representing voltage behaviors in the real-time environment. 


\section{Chapter 3}

\section{OPF-LI with State Calculator}

Many load centers exist in interconnected power systems. A major load center is usually supplied by multiple transmission facilities and generation from external system through several boundary buses. The growth in electric energy consumption in a load center leads to heavy power transfer between the external system and the load area. Based on these properties load centers are likely to experience voltage instability. Monitoring voltage stability at a load center area is an important part of power system voltage stability assessment. VSAI-I and VSAI-II indices are derived for a load bus based on a Thevenin equivalent (TE) which approximate the rest of the power system by a voltage source connected through a Thevenin impedance. In this thesis, a new method, OPF-LI, is developed to extend the voltage stability index from two nodes to multiple nodes model with more accurate estimation.

\subsection{Ward-PV model}

To investigate voltage stability of a load area, details of the load area and generation sources cannot be eliminated. In this project, an extension is proposed to enhance the model using the Ward-PV equivalent system model [15].In the Ward-PV model shown in Figure 3.1, the entire set of buses are partitioned into three subsets: boundary, external and internal buses. External buses are further partitioned into PV and PQ buses. External PQ buses are eliminated while the PV buses are retained. Therefore, the external system is modeled by a set 
of external generators and their (equivalent) transmission lines connecting to the load area through the boundary buses. The topology of the load center is preserved in this equivalent model. Then the reactive power response of the external generators is calculated based on decoupled reactive power flow [15].

The proposed model has the following advantages:

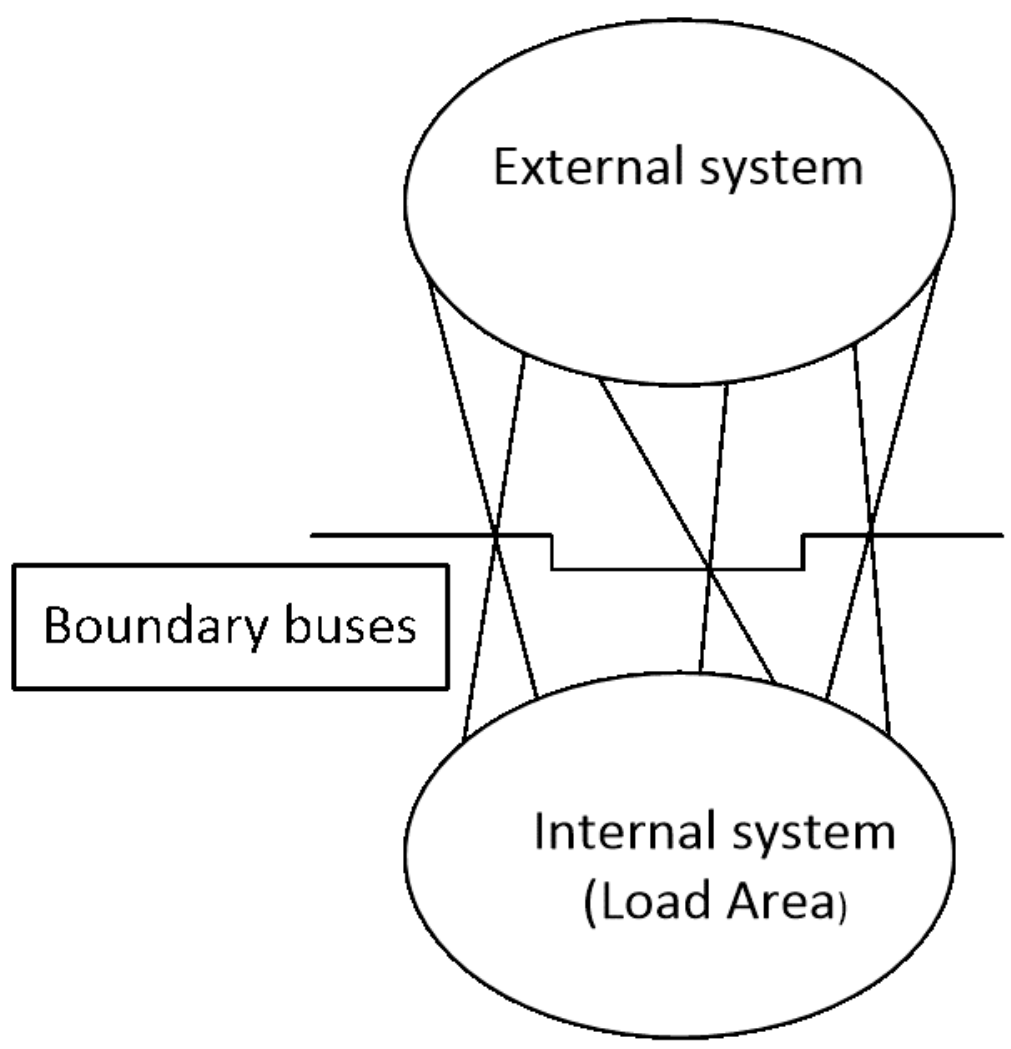

Figure 3.1: Ward-PV model

(1) Modeling a load area fed by several external generators. The effect of external PV buses as well as the detailed model of the load area is preserved.

(2) The Ward-PV equivalent provides reasonably accurate results in terms of real and reactive power flow. 
(3) The large scale of the external system is reduced to a number of tie lines with the generation sources. Hence, it is feasible to compute the voltage stability margin in an on-line environment.

\subsection{OPF based algorithm}

Based on the Ward-PV equivalent system, the maximum allowable loading for the entire load area is given by the optimal solution of a new OPF based model. That is,

(1) The objective of optimization is the load ratio by which the loads is uniformly modified by one parameter $\lambda$. The generator real and reactive power injections are the vector of $P_{g}, Q_{g}$ and the voltage angles and magnitudes are the vector of $\theta$ and $v$.

(2) Objective function: $\max \lambda$

(3) Constraints:

The updated equality constraints:

$$
\begin{aligned}
& P(v, \theta)+\lambda P_{l}-P_{g}=0 \\
& Q(v, \theta)+\lambda Q_{l}-Q_{g}=0
\end{aligned}
$$

The inequality constraints:

$$
\begin{aligned}
& 0 \leq \lambda \geq 10 \\
& \left|S_{b}(\theta, v)\right|-S_{\text {max }} \leq 0 \\
& v_{\text {min }} \leq v \leq v_{\text {max }} \\
& P_{\text {gmin }}, Q_{\text {gmin }} \leq P_{g}, Q_{g} \leq P_{\text {gmax }}, Q_{\text {gmax }}
\end{aligned}
$$


All constraints of the system are associated with characteristics of the base case. Hence, the inequality constraints of external generator injections are identical with those of the base case. The line flow constraints of the fictitious equivalent external lines are approximated based on the power flow results of the equivalent system.

\subsection{OPF-LI with State Calculator}

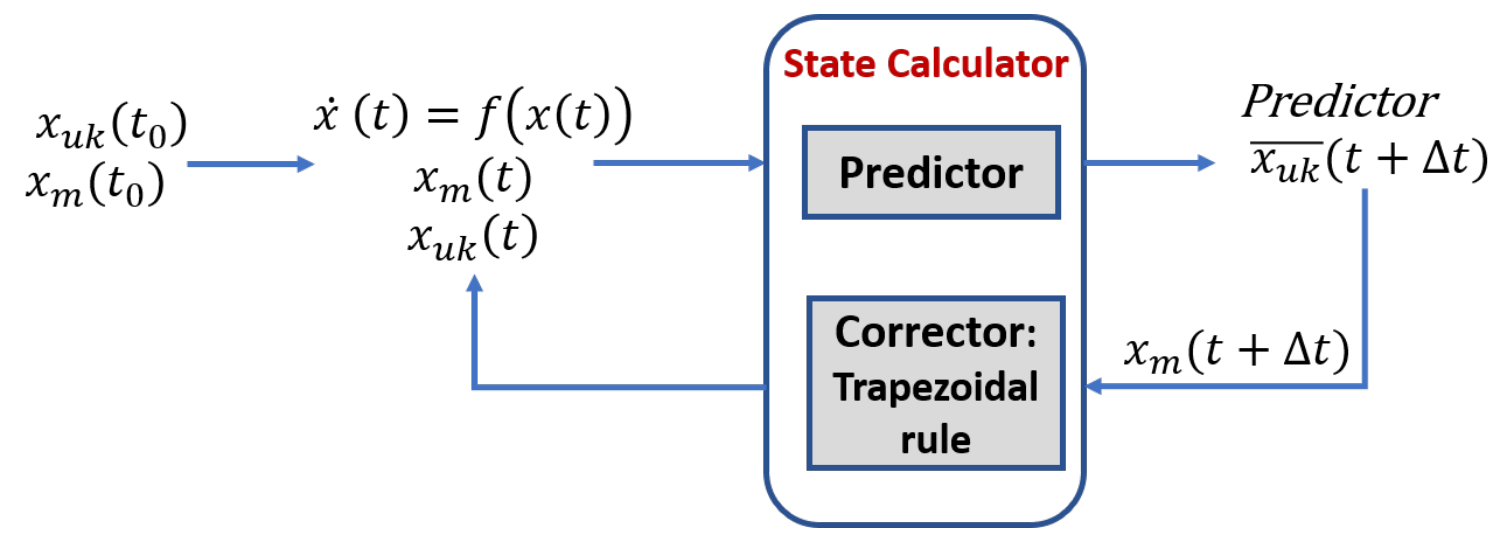

Figure 3.2: The flowchart of State Calculator (SC)

The proposed algorithm OPF-LI uses the network information and PMU data. State Calculator (SC) developed in [14] is able to estimate the unavailable PMU measurement with available PMU data. As shown in Figure 3.2, between two time instant $t$ and $t+\Delta t$, the predictor of the unknown variable $x_{u k}^{-}(t+\Delta t)$ is predicted in terms of measurement $x_{m}(t)$, and the approximate value from last time instant $x_{u k}(t)$. With the measurement $x_{m}(t+\Delta t)$, the unknown variable $x_{u k}(t+\Delta t)$ is corrected by the trapezoidal rule. With the state variables estimated by SC, the generator electrical power output $P_{e i}$ is calculated in (3.7) using 
rotor angle $\delta$, generator dynamic states $E_{q}^{\prime}, E_{q}^{\prime}$, generator terminal voltage $V_{i}$. Since $x^{\prime}=x_{q}^{\prime}$ :

$$
\begin{aligned}
& I_{i}=Y V_{i} \\
& I_{i}=\left(I_{d i}+j I_{d i}\right) e^{-j\left(\delta_{i}-\frac{\pi}{2}\right)} \\
& P_{e i}=E^{\prime}{ }_{d} I_{d i}+E_{q}^{\prime} I_{q i}
\end{aligned}
$$

As the generator states $P_{e}, V$ estimated by $\mathrm{SC}$ with limited PMU data, the initial value for OPF-LI are updated as the time step of simulation advances.

The Ward-PV equivalent system is used to simplify the system topology. Based on the Ward-PV equivalent system and the system states estimated by SC with limited PMU data, the maximum allowable loading for the entire load area is given by the optimal solution of a new Optimal Power Flow (OPF) based model. That is, the objective of optimization is the load ratio by which the loads are uniformly modified by one parameter $\lambda$. Note that with the network reduction, the computational speed for the optimization is high. 


\section{Chapter 4}

\section{OLTC operating control}

Based on the proposed dynamic mechanism of voltage stability, voltage control using OLTC operating principles is proposed. OLTC performs load restoration by adjusting tap positions of transformers automatically. The tap changers monitor the secondary voltage $V_{2}$ by updating the transformer ratio. Because of the mechanical time delay and intentional time delay, OLTC is operating in discrete values as shown in (9).

The stability analysis for power systems using continuous OLTC model is investigated in [9]. With one OLTC in the system, the number of equilibrium points considering the OLTC dynamic model will be two (or less), represented as $n_{10}, n_{20} . n_{20}$ is the unstable equilibrium point, which is also known as the bifurcation point. In case the tap position moves beyond $n_{2} 0, n$ will increase since $\frac{d n}{d t}>0$. However load voltage $V_{2}$ falls down, indicating a voltage collapse in the system. Generalizing the voltage stability analysis with n OLTCs, there can be multiple equilibrium points. It is difficult to calculate the equilibrium points and the voltage stability region. Instead, the dynamic behavior of multiple OLTC is included in the proposed voltage control strategy.

The proposed OLTC operating control is presented below, where $k$ denotes the tap step. 
(1) OLTC Operation

$$
\begin{array}{r}
\operatorname{tap}(k)-\operatorname{tap}(k-1)=0,-1 \text { or } 1 \\
\operatorname{tap}^{-} \leq \operatorname{tap}(k) \leq \operatorname{tap}^{+}
\end{array}
$$

If $V(k)-V_{r e f} \geq \Delta v_{r e f}$,

$$
\operatorname{tap}(k+1)-\operatorname{tap}(k)=1
$$

Else if $V(k)-V_{r e f} \leq-\Delta v_{r e f}$

$$
\operatorname{tap}(k+1)-\operatorname{tap}(k)=-1
$$

Else if $\left|V(k)-V_{r e f}\right|<\Delta v_{r e f}$

$$
\operatorname{tap}(k+1)-\operatorname{tap}(k)=0
$$

(2) OLTC blocking

$$
\begin{gathered}
\text { If } \operatorname{tap}(k)-\operatorname{tap}(k-1)=-1 \quad A N D \quad V(k)-V(k-1) \leq 0 \\
\operatorname{tap}(k+1)=\operatorname{tap}(k)
\end{gathered}
$$

According to the normal operation described in (4.14.3), the tap changer tries to raise the load side voltage to correct the deviation of load voltage from the set point. However, the bus voltage possibly falls due to the limited range of applicability of the OLTC control principle. Utilizing the relation between $\Delta$ tap and $\Delta V$, OLTC blocking control is implemented as described in (4.4). The blocking control is triggered on condition that voltage starts to 
decline with the tap action moving as an attempt to raise the secondary voltage. Therefore, the designated tap changer will be blocked as soon as $\Delta$ tap $<0$ and $\Delta V \leq 0$. This blocking action prevents the operating point from entering the instability region. 


\section{Chapter 5}

\section{Simulation Results}

This section provides the calculation results of the proposed indices on a 179-bus model resembling the structure of WECC. As illustrated in Figure 5.1, the analysis is focused on Area 1 of the system as the load area. The base case load for Area1 is:

$$
\begin{gathered}
P_{L}^{\text {Area } 1}=3.64 \times 10^{4} M W \\
Q_{L}^{\text {Area1 }}=6.66 \times 10^{3} M V A R
\end{gathered}
$$

The computation involved in the algorithms is performed by MATLAB. The commercially available tool, TSAT, is used to validate the stability margin using the dynamic model of the 179-bus system. 


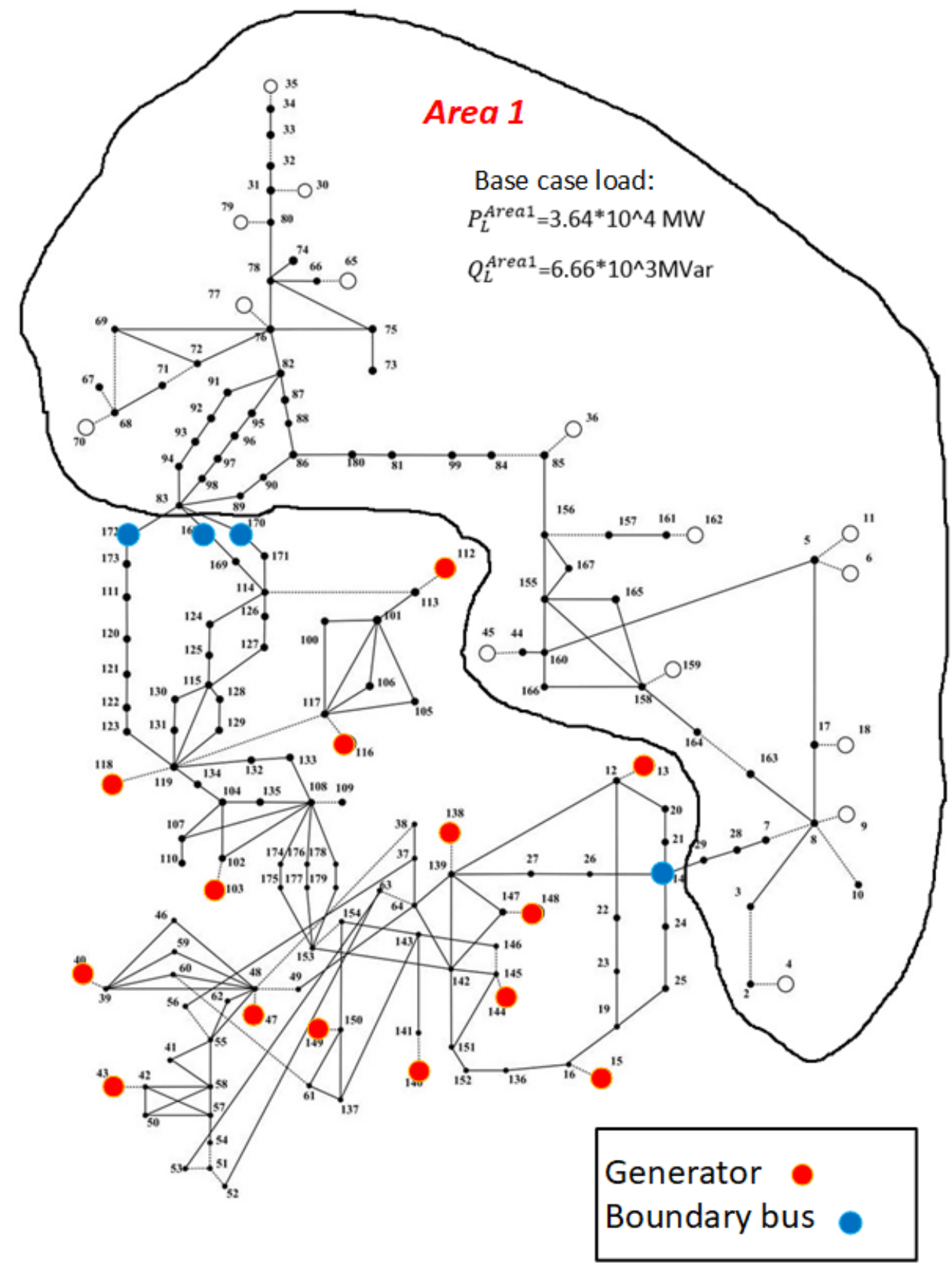

Figure 5.1: Area 1 of WECC 179 bus system 


\subsection{Comparison of VSAI-I and VSAI-II}

The VSAI-I, a version of VSAI based on the steady state model of VSAI in section 2.1, performance is validated by simulation for IEEE 30-bus System test under different scenarios of deteriorating voltage stability conditions.

As shown in Figure 5.2, when the contingencies take place (i.e. when the transmission lines between buses 6,8 and 6,28 are taken out of the system), the voltage magnitudes at the buses decreased, particularly at Bus 8. This is due to the fact that there is only one generator feeding Bus 8 after two lines are de-energized. This is indicated by a significantly increased $V S A I(V S A I 8=0.7159)$ of the affected load bus (Bus 8). Hence the system instability index is high.This information is visualized at the control center giving the operator information about the critical locations for voltage stability and allows the operator to take appropriate actions based on control actions suggested by the tool.
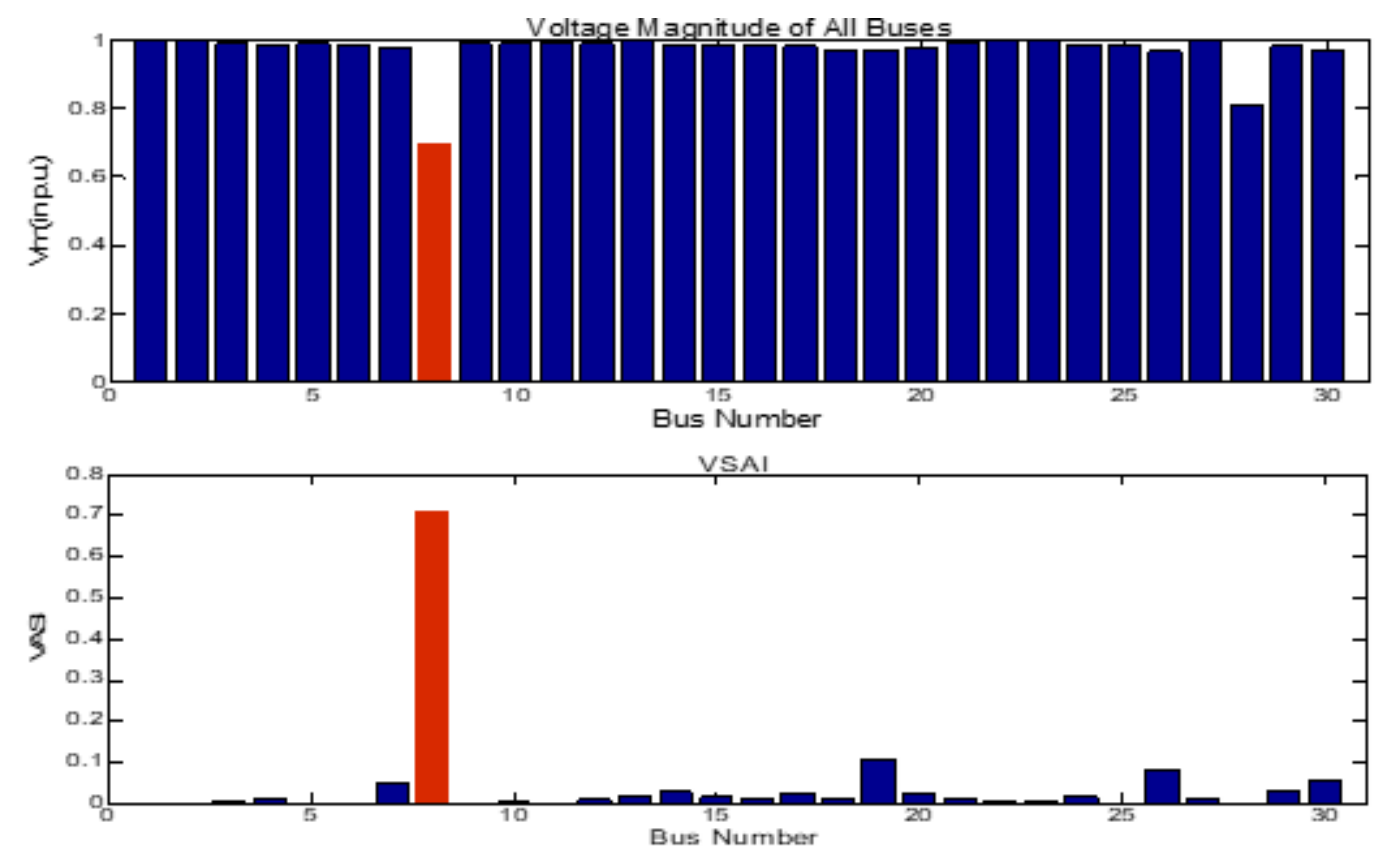

Figure 5.2: VSAI and voltage magnitudes of IEEE 30-bus system after a contingency 
In order to compare the results obtained from VSAI-I and VSAI-II, the The WECC 179 bus system as the test system is used for comparison.

Contingency: Increase load in Area1 to 1.1 times of base case loading.

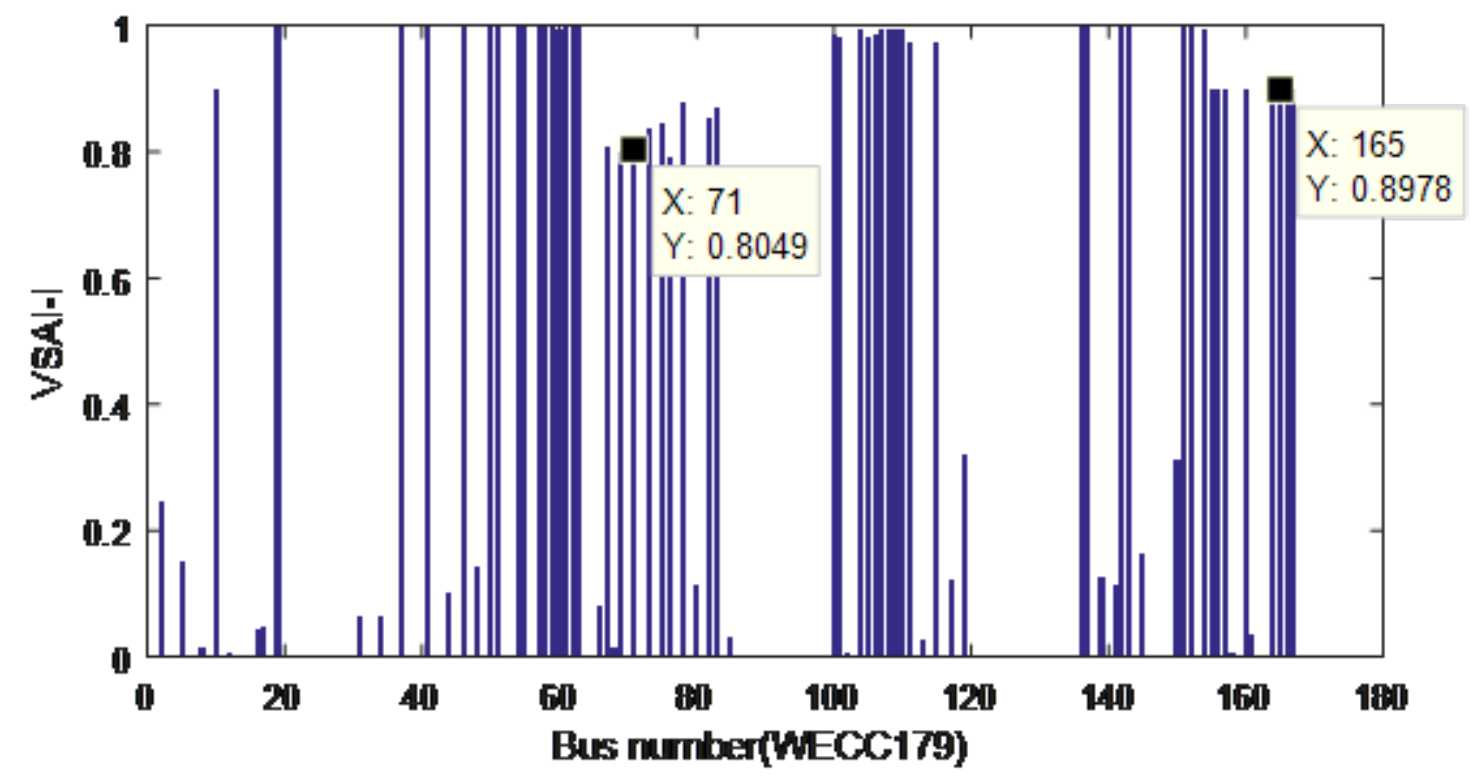

Figure 5.3: VSAI-I of the load buses

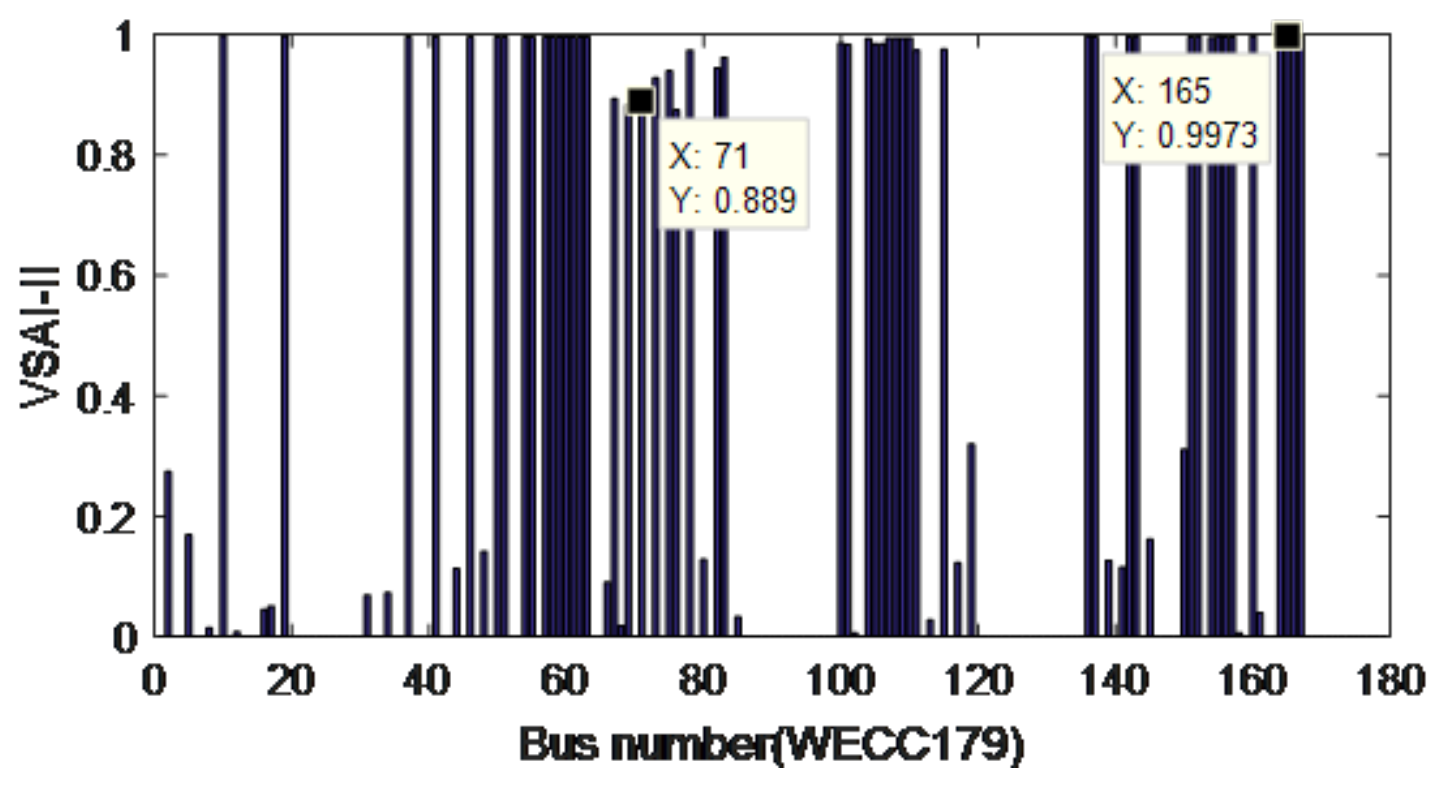

Figure 5.4: VSAI-II of the load buses 
As shown in Figure 5.3, some values of the VSAI-I are equal to 1, indicating the system is close to instability. However, for VSAI-II, most values are equal to 1 indicating that the stability margin is small. For both cases, the results show the system is on the verge of voltage instability. Since no dynamic mechanism is modeled in the VSAI-I index, the system condition reflected by the upper diagram is more stable than that of VSAI-II. That is, VSAI-II is a more realistic indicator of the system operating condition. Note that the base case has a heavy loading condition and, therefore, the stability margin is expected to be small.

\subsection{Validation of OPF-LI with TSAT}

To determine the loading limit of Area 1, shown in Figure 5.1, load in Area 1 ramps up from 10 seconds and stays flat after 30 seconds during the time domain simulation. In the WECC 179 system, load levels at the buses in Area 1 that are connected to large power import/export through tie lines are fixed. TSAT as a time domain simulator is used as the validation tool. The trajectory of voltage magnitude and the absolute value of generator angles are illustrated in Figure 5.5. When load in area 1 is gradually increased to 1.25 times of the base case load, system tends to be stable even though the voltage drops. However, It shows that the voltage profiles are declining, and generators begin to lose syn-

chronism once the load in Area 1 is increased to 1.26 times of the base case. In other words, the loading limits for Area 1 in terms of voltage stability is about 1.25 times of the base case. 


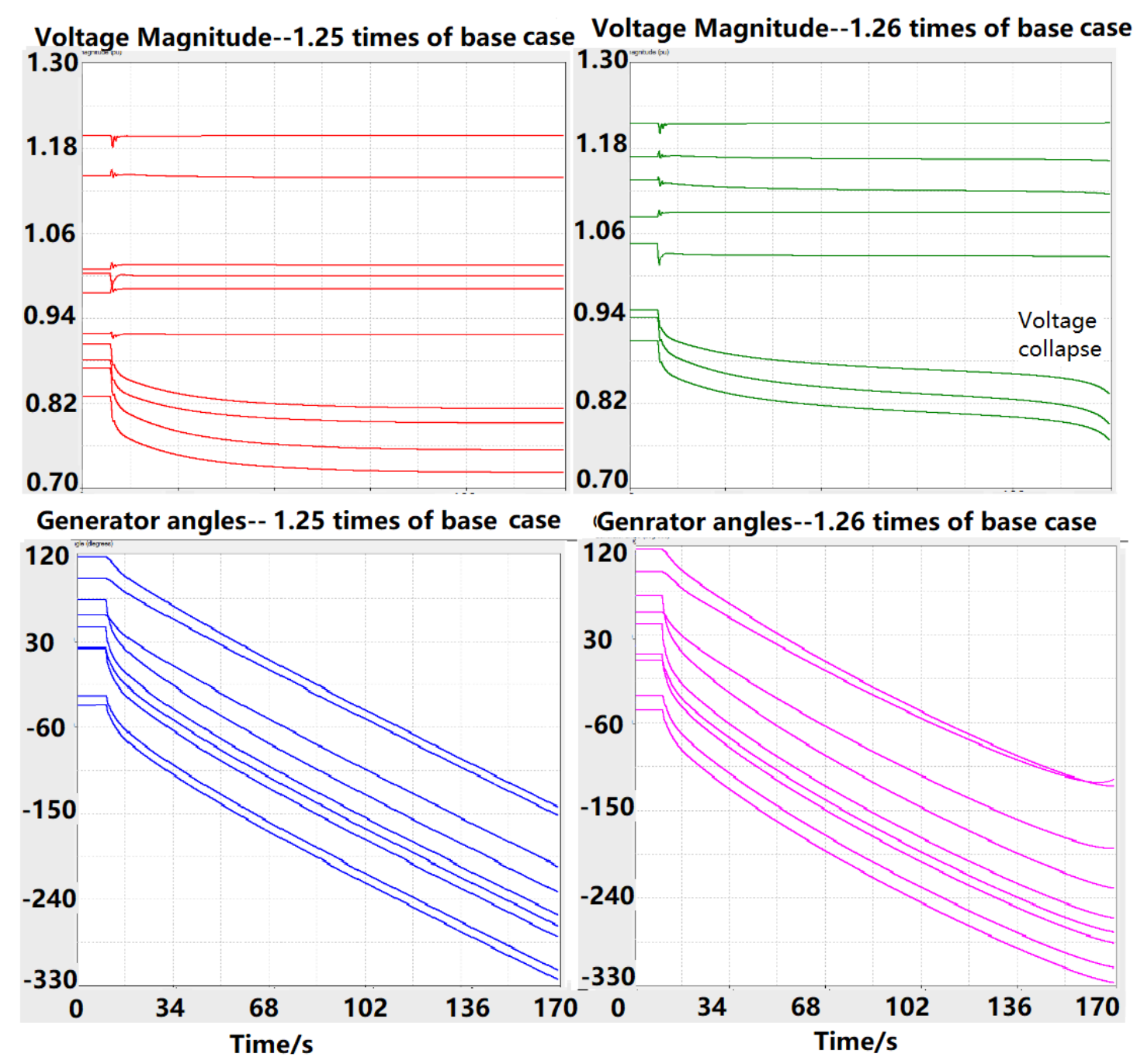

Figure 5.5: System states (Bus voltage and angle) in Area1increased to1.25 or 1.26

The proposed OPF-LI is used to estimate the maximum allowable load of Area 1. As shown in 5.1, the red dots represent the generators from the external system, and blue dots are the boundary bus for Ward-PV. Also, considering the excitation system of the generators, the voltage magnitudes at generator buses in OPF-LI model are fixed. For the OPF-LI algorithm, the load ratio is represented by the optimal value $\lambda$ and the initial value for $\lambda$ is 
1. As shown in Table 5.1, the loading limit of Area 1 estimated by OPF-LI is 1.19 times of the base case.

Table 5.1: Results of OPF-LI.

\begin{tabular}{ccccc}
\hline \multicolumn{5}{c}{ System Status of the estimated point } \\
\cline { 3 - 5 }$\lambda_{\max }$ (Loading limit) & $\lambda-1$ (voltage stability margin) & $P_{g}, Q_{g}$ constraints & Voltage magnitude constraints & $S_{b}$ constraints \\
\hline 1.19 & 0.19 & None & None & Bus26 28,7980,8084 \\
\hline
\end{tabular}

Comparing TSAT with OPF-LI (Fixing V of PV bus), the loading limits derived from TSAT, i.e. 1.25, is a bit higher than that of OPF-LI. The voltages at PV buses in OPF-LI model are fixed during the calculation, which is similar to the function of exciters in the TSAT dynamic model. However, as power flow constraints are not considered during the time domain simulation in TSAT, the voltage stability margins of OPF-LI are more conservative than those of TSAT. To validate VSAI-II in the same scenario, the proposed indices are calculated at each load bus with the load in Area 1 increased to 1.2 times of the base case load. As the results of VSAI-II shown in Figure 5.4, most values equal to 1 indicating that the stability margin is expected to be small when the load in Area 1 reach 1.2 times of the base case. However, it is noted that when the load keeps increasing, the Jacobian matrix is close to being singular and the proposed VSAI-II fails to estimate the indices.

Comparing OPF-LI with VSAI-II, both methods are able to monitor voltage stability using PMU measurements. However, OPF-LI approximates the loading margin directly, while VSAI method assesses the voltage stability of a power system with no indication of the margin. 


\subsection{OPF-LI with SC}

The WECC 179-bus system in Figure 5.1 is used to validate the proposed OPF-LI with SC. The algorithm is implemented in MATLAB. The system has 29 generators, however it is assumed that PMU measurements are only available at 15 generators as presented in Table 5.2. To illustrate the performance of OPF-LI with SC, the following two study case are analyzeds:

Case 1: At $\mathrm{t}=1 \mathrm{~s}$, a three-phase fault at generator bus 18 is applied.The generator is tripped as fault cleared in 4 cycles. The simulation lasts 100 seconds.

Case 2: A three-phase faults at line $82-95$ is applied at $t=1$ second. The fault is cleared in 4 cycles, and the line is de-energized. The simulation lasts 100 seconds.

Table 5.2: PMU location

\begin{tabular}{cc}
\hline Numbers of PMUs & Generators with PMU measurements \\
\hline 15 & $1,2,5,6,7,8,9,10,11,12,15,16,17,19,24$ \\
\hline
\end{tabular}

\subsubsection{Case 1}

Using TSAT time domain simulation results as the synthesized PMU data of the 15 generator buses, the states of the other generators can be estimated for every cycle by the SC. As the results shown in Figure 5.6, it is observed that the system tends to be stable after tripping generator bus 18 .

Incorporating the results of SC, OPF-LI for Area1 is calculated for every 5 seconds, and the results are shown in Figure 5.7. 

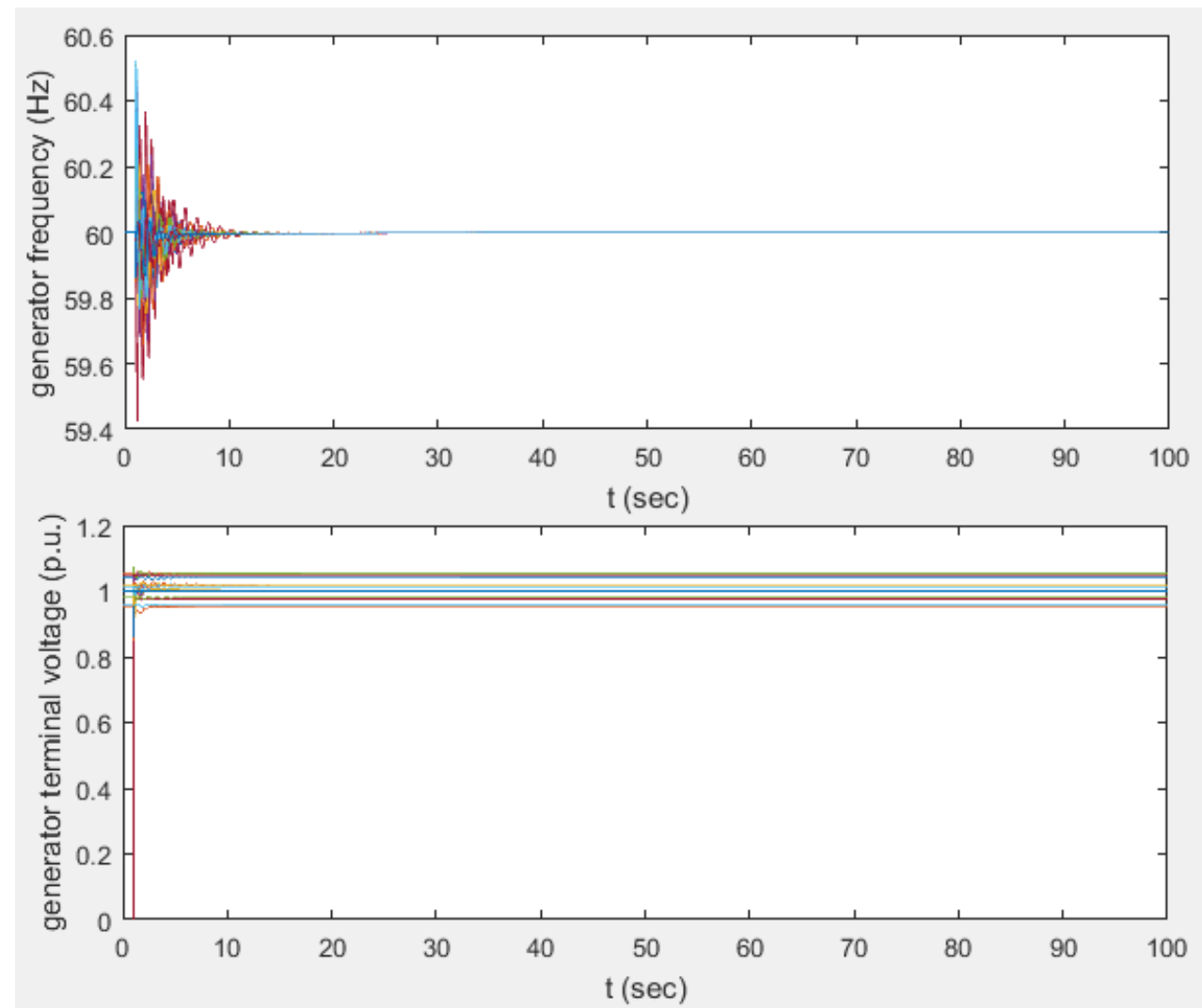

Figure 5.6: State calculator results-Case1

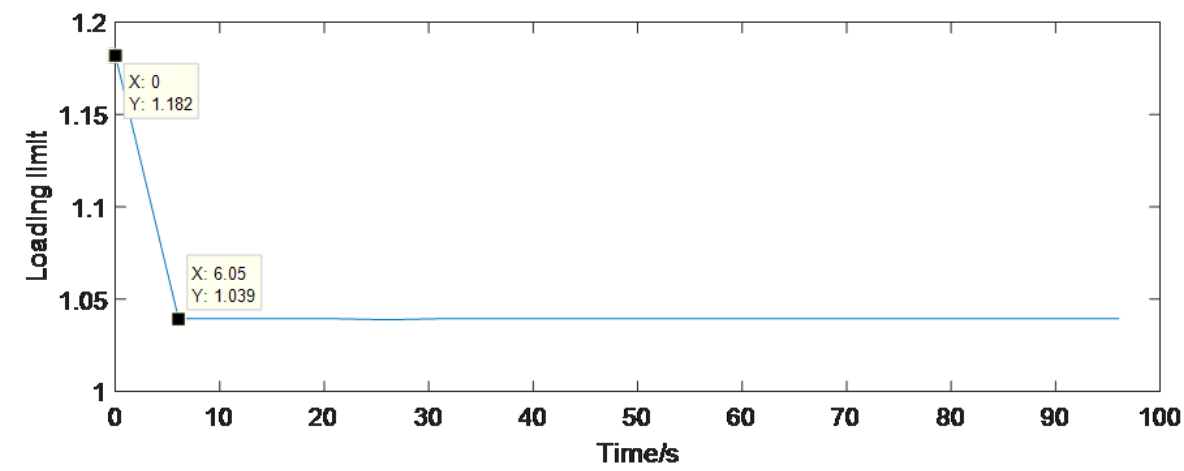

Figure 5.7: The loading limit during the contingency-Case 1

It is observed that the loading limit goes down to 1.039 due to generator tripping. Since the generator at bus 18, located in Area1, is tripped after the fault, the remaining generators, mostly from the external area, increased their output to make up for the lost generation 
causing the available transmission capacity to be reduced. Thus, the loading limit for Area1 decreased after the line fault.

\subsubsection{Case 2}

As the results shown in Figure 5.8, it is observed that the system tends to be stable after tripping line 82-95. Based on the results of SC, the OPF-LI for Area 1 is calculated every 5 seconds.
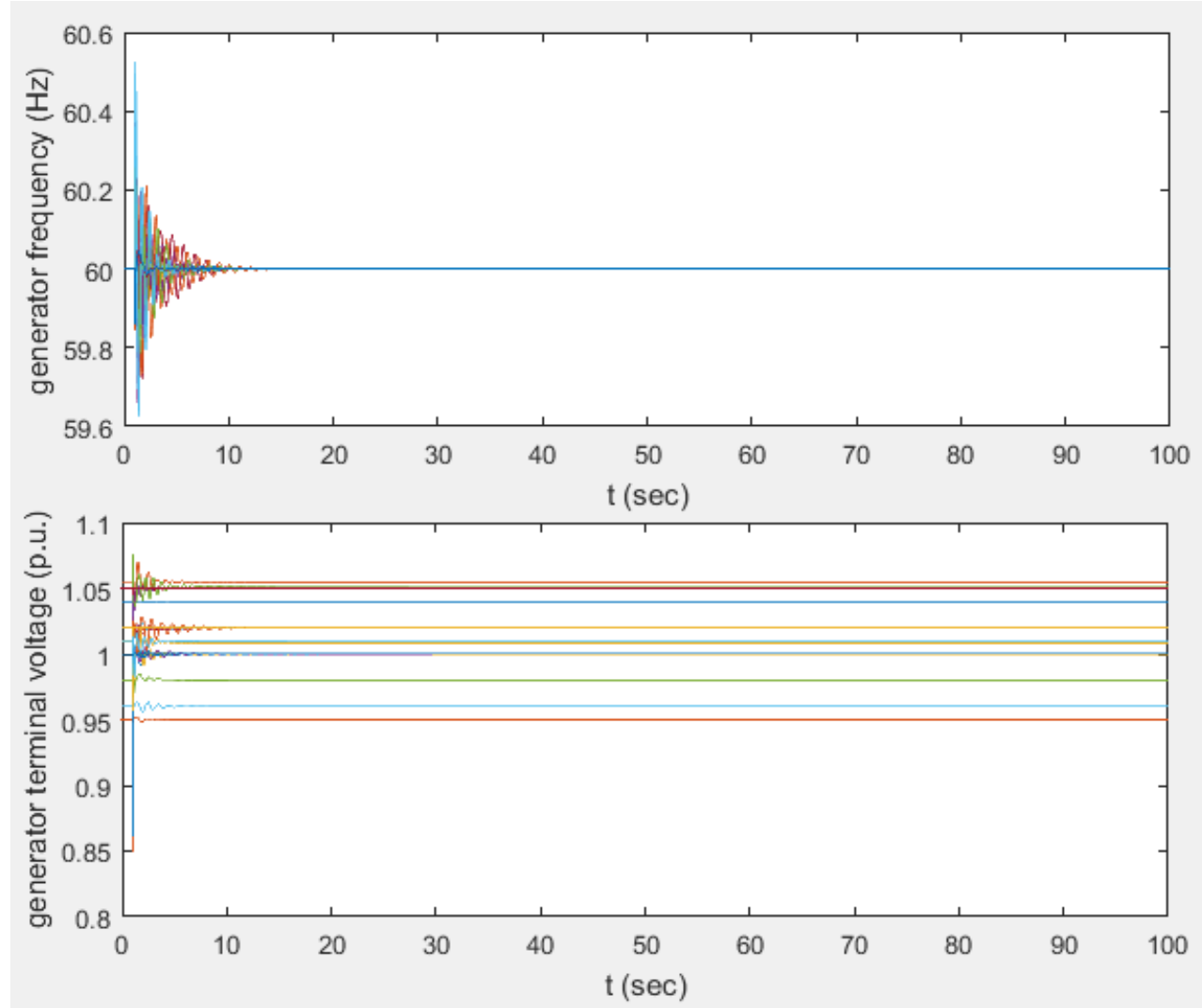

Figure 5.8: State calculator results - Case2 


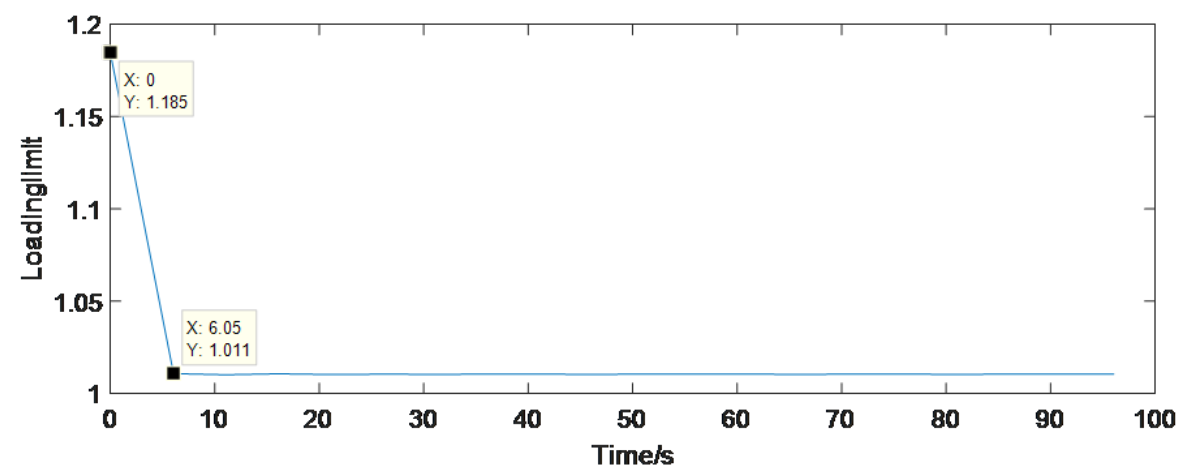

Figure 5.9: The loading limit during the contingency-Case 2

As shown in Figure 5.9, the load margin of Area 1 decreases to 1.039 after the line fault on line 82-95. The Ward-PV equivalent system is updated based on the post-fault system topology. Since a line is tripped, the line flow constraint of line 82-95 no longer applies; furthermore, the line flow for other lines will increase, causing the loading limit to be reduced. Although the system is stable, the reduced loading limit obtained by OPF-LI indicates that the system is stressed after the line fault.

\subsection{OLTC operating control}

The WECC 179 bus system is used as the test system for validation of the proposed OLTC operating control. According to the results of OPF-LI in the simulation, the loading limit of Area 1 is around 1.19. Therefore, assume that the load of Area1 increases to 1.19 times of the base case loading. Meanwhile, the actions of OLTCs are triggered by the voltage deviation.

As shown in Figure 5.10 and Figure 5.11:

Blue curve: the voltage reference at each bus.

Red curve: the voltage profile at the beginning of tap operation. 
Green curve: the voltage profile after tap operations.

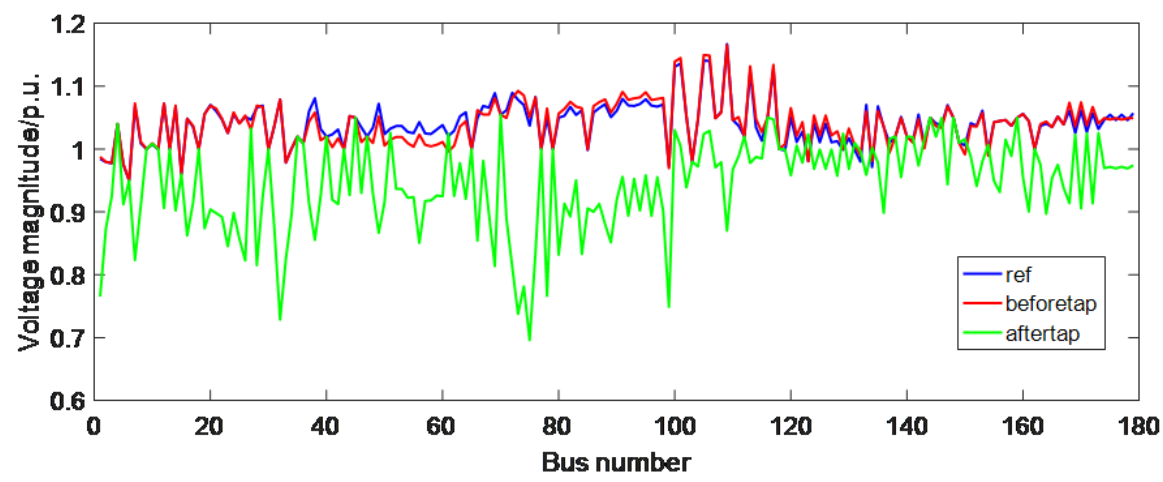

Figure 5.10: The comparison of voltage profiles - without OLTC blocking control

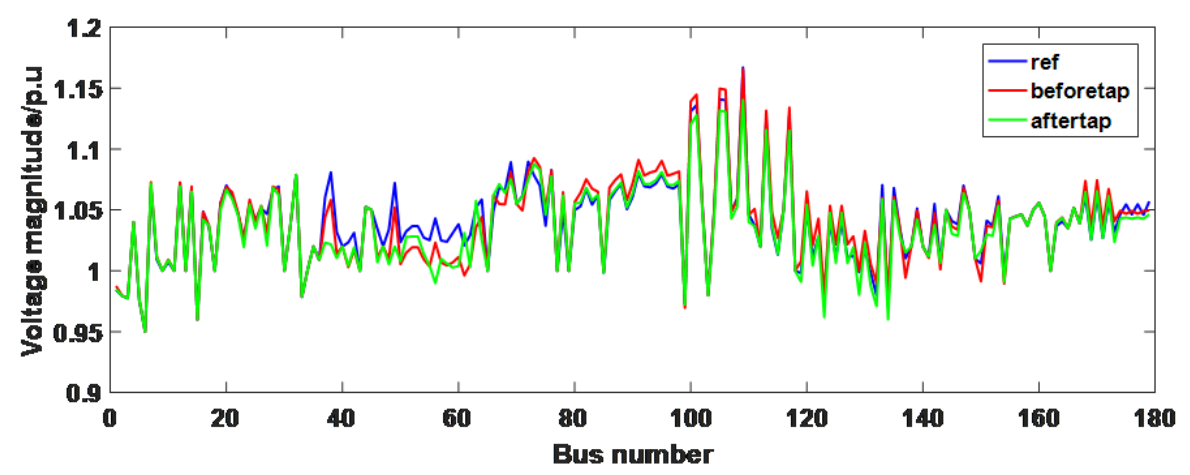

Figure 5.11: The comparison of voltage profiles - with OLTC blocking control

Figure 5.10 indicates that the voltage profile represented by the green curve is not improved after the tap operation. It is observed that the voltage at most buses drops in comparison with the red curve. Without OLTC blocking, the tap changing causes the voltage drops when the operating point falls out of the recovery region. In this case, some tap changers are blocked at the limits when the tap ratio reaches the upper or lower limits, so that the system condition does not deteriorate. As shown by the green curve in Figure 5.11, the voltage profile is improved due to OLTC blocking control. Along with the operation of the proposed OLTC control, OLTCs located at transformer buses 46, 47, 48 ,50, 53 are blocked 
based on the criteria discussed in Section IV. By doing so, the dynamic behavior of OLTC is improved and the system condition is restored without a voltage collapse. 


\section{Chapter 6}

\section{Conclusions and Future Works}

This paper provides new computational techniques for smart grid voltage stability assessment and control. This work includes three parts:

(1) By incorporating the dynamic mechanisms into voltage stability assessment, VSAI-II is able to detect voltage instability of the system. The indices indicate the critical locations in the system.

(2) Utilizing SC, OPF-LI monitors the voltage stability margin of the load center with limited PMU measurements.

(3) (3) According to the dynamic features of OLTCs, the novel OLTC blocking control is developed to prevent the system operating point from falling out of voltage recovery region.

For the future work, the proposed OLTC control should be incorporated in the integer programming-based method in order to determine the optimal OLTC actions at multiple locations in a real time environment. Also, because of the time sensitivity of online application in a control center, it is desirable to improve the computational speed of the proposed OPF based algorithm. For the planning purpose, the proposed method provides locations where voltage stability can be improved through transmission enhancement. 


\section{Bibliography}

[1] Venkataramana Ajjarapu and Colin Christy. The continuation power flow: a tool for steady state voltage stability analysis. IEEE transactions on Power Systems, 7(1):416423, 1992.

[2] Chumki Basu, Ankit Agrawal, Jagabondhu Hazra, Ashok Kumar, Deva P Seetharam, Jean Béland, Sébastien Guillon, Innocent Kamwa, and Claude Lafond. Understanding events for wide-area situational awareness. In ISGT 2014, pages 1-5. IEEE, 2014.

[3] Saugata Swapan Biswas and Anurag K Srivastava. Voltage stability monitoring in power systems, January 23 2018. US Patent 9,876,352.

[4] Ruisheng Diao, Kai Sun, Vijay Vittal, Robert J O’Keefe, Michael R Richardson, Navin Bhatt, Dwayne Stradford, and Sanjoy K Sarawgi. Decision tree-based online voltage security assessment using pmu measurements. IEEE Transactions on Power systems, 24(2):832-839, 2009.

[5] N Flatabo, R Ognedal, and T Carlsen. Voltage stability condition in a power transmission system calculated by sensitivity methods. IEEE Transactions on Power Systems, 5(4):1286-1293, 1990.

[6] Mevludin Glavic and Thierry Van Cutsem. Wide-area detection of voltage instability from synchronized phasor measurements. part i: Principle. IEEE Transactions on Power Systems, 24(3):1408-1416, 2009.

[7] Oscar Gómez and Mario A Rios. Inter-area stability prediction index based on phasorial 
measurement. In 2012 IEEE PES Innovative Smart Grid Technologies (ISGT), pages 1-8. IEEE, 2012.

[8] Fengkai Hu, Kai Sun, Alberto Del Rosso, Evangelos Farantatos, and Navin Bhatt. Measurement-based real-time voltage stability monitoring for load areas. IEEE Transactions on Power Systems, 31(4):2787-2798, 2015.

[9] C-C Liu and Khoi T Vu. Analysis of tap-changer dynamics and construction of voltage stability regions. IEEE Transactions on circuits and systems, 36(4):575-590, 1989.

[10] Iraj Rahimi Pordanjani, Yunfei Wang, and Wilsun Xu. Identification of critical components for voltage stability assessment using channel components transform. IEEE Transactions on Smart Grid, 4(2):1122-1132, 2013.

[11] Thierry Van Cutsem and Costas Vournas. Voltage stability of electric power systems. Springer Science \& Business Media, 2007.

[12] Khoi Vu, Miroslav M Begovic, Damir Novosel, and Murari Mohan Saha. Use of local measurements to estimate voltage-stability margin. IEEE Transactions on Power Systems, 14(3):1029-1035, 1999.

[13] Khoi T Vu and Chen-Ching Liu. Dynamic mechanisms of voltage collapse. Systems $\&$ control letters, 15(4):329-338, 1990.

[14] Guanqun Wang, Chen-Ching Liu, Navin Bhatt, Evangelos Farantatos, and Mahendra Patel. Observability of nonlinear power system dynamics using synchrophasor data. International Transactions on Electrical Energy Systems, 26(5):952-967, 2016.

[15] Felix Fulih $\mathrm{Wu}$ and A Monticelli. Critical review of external network modelling for online security analysis. International Journal of Electrical Power $\& 3$ Energy Systems, $5(4): 222-235,1983$. 
[16] Ce Zheng, Vuk Malbasa, and Mladen Kezunovic. Regression tree for stability margin prediction using synchrophasor measurements. IEEE Transactions on Power Systems, 28(2):1978-1987, 2012. 